\title{
Fluorescence control through multiple interference mechanisms
}

\author{
E. Paspalakis, ${ }^{1}$ C. H. Keitel, ${ }^{2,3}$ and P. L. Knight ${ }^{1}$ \\ ${ }^{1}$ Optics Section, Blackett Laboratory, Imperial College, London SW7 2BZ, United Kingdom \\ ${ }^{2}$ Theoretische Quantendynamik, Fakultät für Physik, Universität Freiburg, Hermann-Herder-Strasse 3, D-79104 Freiburg, Germany \\ ${ }^{3}$ Institut für Theoretische Physik, Universität Innsbruck, Technikerstrasse 25/2, A-6020 Innsbruck, Austria
}

(Received 26 May 1998)

\begin{abstract}
We discuss the spontaneous emission from a coherently prepared and microwave-driven doublet of potentially closely spaced excited states to a common ground level. Multiple interference mechanisms are identified that may lead to fluorescence inhibition in well-separated regions of the spectrum or act jointly in canceling the spontaneous emission. In addition to phase-independent quantum interferences due to combined absorptions and emissions of driving field photons, we distinguish two competing phase-dependent interference mechanisms as means of controlling the fluorescence. The indistinguishable quantum paths may involve the spontaneous emission from the same state of the doublet, originating from the two different components of the initial coherent superposition. Alternatively the paths involve a different spontaneous photon from each of two decaying states, necessarily with the same polarization. This makes these photons indistinguishable in principle within the uncertainty of the two decay rates. The phase dependence arises for both mechanisms because the interfering paths differ by an unequal number of stimulated absorptions and emissions of the microwave field photons. [S1050-2947(98)03011-X]

PACS number(s): 42.50.Ct, 32.80.Qk, 42.50.Lc, 42.50.Ar
\end{abstract}

\section{INTRODUCTION}

The study of spontaneous emission and the various means by which it may be modified and controlled has been an active area of quantum optics for many years. As fluorescence arises from the interaction of the atomic system with the environmental modes, the most obvious mechanism for control is to place the atoms in "colored" (frequencydependent) reservoirs [1]. This can be achieved by employing, for example, atoms in microcavities [2] or by placing them near the edge of photonic band gaps [3]. For atomic media in free space, quantum interferences have become the most significant mechanism for modifying spontaneous emission. This was suggested in the early 1970s when Agarwal [4] showed, for an initially prepared degenerate V-type three-level atom in free space, that the fluorescence spectrum is modified due to interference and that population trapping occurs [5]. This was followed by a number of papers in the late 1970s and early 1980s on such interferences [6]. Recently, with the need for efficient fluorescence control to enable such effects as lasing without inversion [7] and quantum information processing [8] to be realized, much attention has once more been focused on spontaneous emission dynamics from multilevel atoms [9-15]. In a recent article [16], two of us have proposed a two-color coherent "phase" control scheme [17] for controlling spontaneous emission in a four-level atom. Phenomena such as extreme spectral narrowing and partial and total cancellation of fluorescence decay were predicted for specific values of the atomic parameters and the lasers phase difference. Moreover, the spectrum was shown to be controlled very effectively and easily by changing the phase difference of the two lasers used for the excitation.

In this article, using a three-level V-type atom, we discuss a further scheme that offers much promise for controlling spontaneous emission spectra using the phase difference, this time of two successively applied coherent fields between the decaying excited doublet states. The proposed scheme is related to other schemes of coherent control, in particular the well-known "pump-dump" scheme of atomic (molecular) ionization (dissociation) [17], the coherently driven threelevel $\Lambda$-type atom of Martinez et al. [18], and (not withstanding the involvement of a photonic band-gap material) the scheme of Quang et al. [19]. The main finding in this article is the identification of various competing interference mechanisms leading to phase sensitive means for controlling the spontaneous emission spectra. If we suppose that only one of the two excited states decays and that the atom is initially prepared in that decaying state, then phase insensitive interfering paths arise that lead to the cancellation of a specific fluorescence mode, as explained by Knight [6] and Zhu et al. [12]. If instead both states are initially prepared in a coherent superposition and the driving field is weak, then the spectrum may acquire a Fano-type profile. Furthermore, phase-sensitive interference occurs and the spectrum can be controlled via this phase difference. In addition, if both excited states decay such that the emitted spontaneous photons may not be distinguishable, even in principle, then further phase-sensitive interfering paths via different spontaneous channels arise, similar to those pointed out within a driven $\Lambda$-type atom by Martinez et al. [18]. We discuss each kind of interference separately by identifying the appropriate paths and discuss the role of their coexistence within a single scheme. In particular we find that both phase-dependent interference mechanisms may be destructive simultaneously and thus lead to spontaneous emission cancellation in different parts of the spectrum. In addition, fluorescence inhibition by one mechanism may also be enhanced due to the presence of the other. An alternative explanation of the phasedependent interferences is also given in the dressed-state picture. In particular we find, for an appropriate phase difference, that the atom can be prepared in a single decoupled 


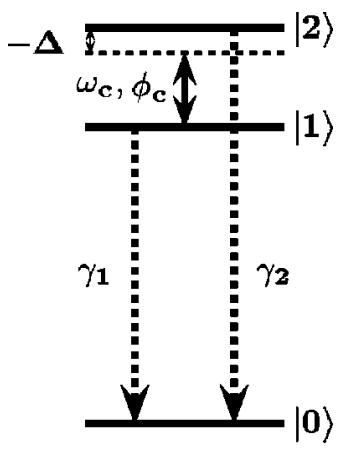

(a)

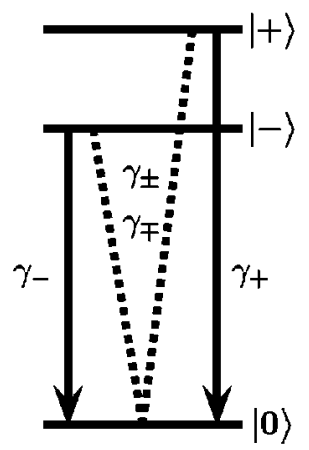

(b)

FIG. 1. System under consideration. (a) Bare-state representation: The two excited states $|1\rangle,|2\rangle$ are coupled by a microwave field with frequency $\omega_{c}$ and phase $\phi_{c}$ and both decay spontaneously to a common ground state $|0\rangle$. (b) Dressed-state representation with dressed decay rates to the ground state $\gamma_{+}$and $\gamma_{-}$(solid lines) and relaxation among the dressed states $\gamma_{\mp}$ from $|-\rangle$ to $|+\rangle$ and $\gamma_{ \pm}$in the opposite direction (dashed lines), which arises from a coupling of the transitions of both dressed states to the ground state.

dressed state in which total cancellation of the fluorescence associated with the other (unpopulated) dressed state occurs.

This paper is organized as follows. In Sec. II we present the atomic model and the basic dynamic equations in the bare-state basis. We solve these equations for the various situations of interest and present, in certain cases, analytical phase-dependent formulas for the time evolution of the population and the spontaneous emission spectrum of this atom. In Sec. III we present and analyze our basic results and in particular discuss the situations where each of the various interference mechanisms becomes significant and dominates. An alternative analysis using the dressed-state basis is given in Sec. IV. Finally, we conclude in Sec. V.

\section{ATOMIC MODEL IN BARE STATES}

The atomic model considered here is shown in Fig. 1. A three level V-type atom is initially prepared in a superposition of the two upper levels by a pump field such that

$$
|\psi(t=0)\rangle=e^{i \phi_{p}} \sin \theta|1,\{0\}\rangle+\cos \theta|2,\{0\}\rangle,
$$

where $\phi_{p}$ is the phase of the pump field. At time $t=0$ this atom starts to interact with a microwave field of frequency $\omega_{c}$ and phase $\phi_{c}$ that couples the two upper levels. We allow both upper states to decay spontaneously to the lower state. Here we consider only the case of a square pulse of the microwave field. This atomic system has been used as a model system for the proposal of the quantum-beat laser [20]. The dynamics of the system can be described using the Schrödinger equation. Then the wave function of the system at time $t$ can be expressed in terms of the state vectors as

$$
|\psi(t)\rangle=a_{1}(t)|1,\{0\}\rangle+a_{2}(t)|2,\{0\}\rangle+\sum_{\mathbf{k}} a_{\mathbf{k}}(t)|0,\{\mathbf{k}\}\rangle,
$$

where $\mathbf{k}$ denotes both the momentum vector and the polarization of the emitted photon. The Hamiltonian of the system in the interaction representation is given by

$$
H_{\text {int }}=H_{\text {field }}+H_{\text {vacuum }} \text {, }
$$

where

$$
\begin{gathered}
H_{\text {field }}=\Omega e^{i \Delta t+i \phi_{c}}|1\rangle\langle 2|+\text { H.c., } \\
H_{\text {vacuum }}=\sum_{\mathbf{k}} g_{1 \mathbf{k}} e^{-i\left(\omega_{\mathbf{k}}-\omega_{10}\right) t}|1\rangle\langle\mathbf{k}| \\
\\
+\sum_{\mathbf{k}} g_{2 \mathbf{k}} e^{-i\left(\omega_{\mathbf{k}}-\omega_{20}\right) t}|2\rangle\langle\mathbf{k}|+\text { H.c. }
\end{gathered}
$$

We substitute this Hamiltonian into the Schrödinger equation and obtain the following set of equations after the rotatingwave approximation is carried out:

$$
\begin{gathered}
i \dot{a}_{1}(t)=\Omega e^{i \Delta t+i \phi_{c}} a_{2}(t)+\sum_{\mathbf{k}} g_{1 \mathbf{k}} a_{\mathbf{k}}(t) e^{-i\left(\omega_{\mathbf{k}}-\omega_{10}\right) t}, \\
i \dot{a}_{2}(t)=\Omega e^{-i \Delta t-i \phi_{c}} a_{1}(t)+\sum_{\mathbf{k}} g_{2 \mathbf{k}} a_{\mathbf{k}}(t) e^{-i\left(\omega_{\mathbf{k}}-\omega_{20}\right) t}, \\
i \dot{a}_{\mathbf{k}}(t)=g_{\mathbf{k} 1} a_{1}(t) e^{i\left(\omega_{\mathbf{k}}-\omega_{10}\right) t}+g_{\mathbf{k} 2} a_{2}(t) e^{i\left(\omega_{\mathbf{k}}-\omega_{20}\right) t} .
\end{gathered}
$$

Here $\Omega$ is the Rabi frequency, which is considered real for convenience in our problem, and $\Delta \equiv \omega_{c}-\omega_{21}$ represents the microwave field detuning. The notation $\omega_{a b}=\omega_{a}-\omega_{b}$ is used throughout this work. We proceed by performing a formal time integration of Eq. (8) and substitute the result into Eqs. (6) and (7) to obtain

$$
\begin{aligned}
i \dot{a}_{1}(t)= & \Omega e^{i \Delta t+i \phi_{c}} a_{2}(t) \\
& -i \int_{0}^{t} d t^{\prime} a_{1}\left(t^{\prime}\right) \sum_{\mathbf{k}}\left|g_{\mathbf{k} 1}\right|^{2} e^{-i\left(\omega_{\mathbf{k}}-\omega_{10}\right)\left(t-t^{\prime}\right)} \\
& -i \int_{0}^{t} d t^{\prime} a_{2}\left(t^{\prime}\right) \sum_{\mathbf{k}} g_{1 \mathbf{k}} g_{\mathbf{k} 2} e^{-i \omega_{\mathbf{k}}\left(t-t^{\prime}\right)+i \omega_{10} t-i \omega_{20} t^{\prime}},
\end{aligned}
$$




$$
\begin{aligned}
i \dot{a}_{2}(t)= & \Omega e^{-i \Delta t-i \phi_{c}} a_{1}(t) \\
& -i \int_{0}^{t} d t^{\prime} a_{2}\left(t^{\prime}\right) \sum_{\mathbf{k}}\left|g_{\mathbf{k} 2}\right|^{2} e^{-i\left(\omega_{\mathbf{k}}-\omega_{20}\right)\left(t-t^{\prime}\right)} \\
& -i \int_{0}^{t} d t^{\prime} a_{1}\left(t^{\prime}\right) \sum_{\mathbf{k}} g_{\mathbf{k} 1} g_{2 \mathbf{k}} e^{-i \omega_{\mathbf{k}}\left(t-t^{\prime}\right)+i \omega_{20} t-i \omega_{10} t^{\prime}} .
\end{aligned}
$$

Once the Markov approximation is carried out within the Weisskopf-Wigner theory [4], Eqs. (9) and (10) reduce to the form (after the assumption $\omega_{21} \ll \omega_{10}, \omega_{20}$ is taken into account)

$\dot{a}_{1}(t)=-\frac{\gamma_{1}}{2} a_{1}(t)-\left(i \Omega e^{i \Delta t+i \phi_{c}}+p \frac{\sqrt{\gamma_{1} \gamma_{2}}}{2} e^{-i \omega_{21} t}\right) a_{2}(t)$,

$\dot{a}_{2}(t)=-\left(i \Omega e^{-i \Delta t-i \phi_{c}}+p \frac{\sqrt{\gamma_{1} \gamma_{2}}}{2} e^{i \omega_{21} t}\right) a_{1}(t)-\frac{\gamma_{2}}{2} a_{2}(t)$.

Here $\gamma_{m} \equiv 2 \pi\left|g_{\mathbf{k} m}\right|^{2} D\left(\omega_{m 0}\right)(m=1,2)$ and the radiative shifts, which are related to the Lamb shift, will be omitted in this approach. The term $p\left(\sqrt{\gamma_{1} \gamma_{2}} / 2\right) e^{ \pm i \omega_{21} t}$ is a common term that arises if quantum interference from both spontaneous emission channels from the two closely spaced upper levels is involved [4]. The parameter $p$ denotes the alignment of the two matrix elements and is defined as $p$ $\equiv \vec{\mu}_{20} \cdot \vec{\mu}_{01} /\left|\vec{\mu}_{20}\right|\left|\vec{\mu}_{01}\right|$. For orthogonal matrix elements this yields $p=0$ (no interference) and for parallel matrix elements we obtain $p=1$ (maximum interference). In the language of quantum pathway interference, different pathways involving different spontaneously emitted photons may be indistinguishable, even in principle, only if the corresponding transitions give rise to photons of identical polarization. This means that the associated dipoles have to be parallel. In the language of off-diagonal couplings, those extra terms in $p$ may arise only if both dipoles involved interact with the same modes of the vacuum. Since the time dependence of this term is $e^{ \pm i \omega_{21} t}$ this term can be omitted from these equations only in the case that the energy difference of the two upper levels $\omega_{21}$ is larger than the decay rates $\gamma_{1}, \gamma_{2}$ [14]. However, for a moment, let us omit these interference terms by setting $p=0$ in order to obtain analytical solutions of these equations. We will return to the importance of such an interference in the calculation of the spectrum. Thus, without the contributions from the $p$ terms in Eqs. (11) and (12), we obtain

$$
\begin{gathered}
\dot{b}_{1}(t)=-\frac{\gamma_{1}}{2} b_{1}(t)-i \Omega e^{i \phi_{c}} b_{2}(t), \\
\dot{b}_{2}(t)=-i \Omega e^{-i \phi_{c}} b_{1}(t)+\left(i \Delta-\frac{\gamma_{2}}{2}\right) b_{2}(t), \\
\dot{b}_{\mathbf{k}}(t)=-i g_{\mathbf{k} 1} b_{1}(t) e^{i\left[\delta_{\mathbf{k}}+(1 / 2) \omega_{21}\right] t} \\
-i g_{\mathbf{k} 2} b_{2}(t) e^{i\left[\delta_{\mathbf{k}}-(1 / 2) \omega_{21}-\Delta\right] t} .
\end{gathered}
$$

Here we define $b_{1}(t) \equiv a_{1}(t), \quad b_{2}(t) \equiv a_{2}(t) e^{i \Delta t}, \quad b_{\mathbf{k}}(t)$ $\equiv a_{\mathbf{k}}(t)$, and $\delta_{\mathbf{k}} \equiv \omega_{\mathbf{k}}-\left(\omega_{10}+\omega_{20}\right) / 2$.

Equations (13) and (14) have the following solutions if $\lambda_{1} \neq \lambda_{2}$ :

$$
b_{1}(t)=C_{1} e^{\lambda_{1} t}+C_{2} e^{\lambda_{2} t}, \quad b_{2}(t)=C_{1}^{\prime} e^{\lambda_{1} t}+C_{2}^{\prime} e^{\lambda_{2} t}
$$

where

$$
\begin{aligned}
\lambda_{1,2}= & \frac{i \Delta}{2}-\frac{\gamma_{1}+\gamma_{2}}{4} \pm \frac{i}{2}\left\{4\left[\Omega^{2}-\left(i \Delta-\frac{\gamma_{2}}{2}\right) \frac{\gamma_{1}}{2}\right]\right. \\
& \left.-\left(i \Delta-\frac{\gamma_{1}+\gamma_{2}}{2}\right)^{2}\right\}^{1 / 2}, \\
C_{1}= & \frac{1}{\lambda_{2}-\lambda_{1}}\left[\left(\lambda_{2}+\frac{\gamma_{1}}{2}\right) \sin \theta+i \Omega e^{i \delta \phi} \cos \theta\right], \\
C_{2}= & \frac{1}{\lambda_{1}-\lambda_{2}}\left[\left(\lambda_{1}+\frac{\gamma_{1}}{2}\right) \sin \theta+i \Omega e^{i \delta \phi} \cos \theta\right], \\
C_{1}^{\prime}= & \frac{1}{\lambda_{2}-\lambda_{1}}\left[\left(\lambda_{2}+\frac{\gamma_{2}}{2}-i \Delta\right) \cos \theta+i \Omega e^{-i \delta \phi} \sin \theta\right], \\
C_{2}^{\prime}= & \frac{1}{\lambda_{1}-\lambda_{2}}\left[\left(\lambda_{1}+\frac{\gamma_{2}}{2}-i \Delta\right) \cos \theta+i \Omega e^{-i \delta \phi} \sin \theta\right] .
\end{aligned}
$$

Here we define the relative phase between the pump and coupling fields as $\delta \phi \equiv \phi_{c}-\phi_{p}$, which as we will show later has a crucial role in the behavior of the system. Also, for convenience, we have set arbitrarily the pump field's phase to zero. So the above phase difference simply reduces to the phase of the coupling field. Using Eq. (15) and solutions (16) we obtain

$$
\begin{aligned}
b_{\mathbf{k}}(t)=- & \frac{g_{\mathbf{k} 1} C_{1}}{\delta_{\mathbf{k}}+\frac{\omega_{21}}{2}-i \lambda_{1}}\left[e^{i\left(\delta_{\mathbf{k}}+\omega_{21} / 2-i \lambda_{1}\right) t}-1\right] \\
- & \frac{g_{\mathbf{k} 1} C_{2}}{\delta_{\mathbf{k}}+\frac{\omega_{21}}{2}-i \lambda_{2}}\left[e^{i\left(\delta_{\mathbf{k}}+\omega_{21} / 2-i \lambda_{2}\right) t}-1\right] \\
- & \frac{g_{\mathbf{k} 2} C_{1}^{\prime}}{\delta_{\mathbf{k}}-\frac{\omega_{21}}{2}-\Delta-i \lambda_{1}}\left[e^{i\left(\delta_{\mathbf{k}}-\omega_{21} / 2-\Delta-i \lambda_{1}\right) t}-1\right] \\
- & \frac{g_{\mathbf{k} 2} C_{2}^{\prime}}{\delta_{\mathbf{k}}-\frac{\omega_{21}}{2}-\Delta-i \lambda_{2}}\left[e^{i\left(\delta_{\mathbf{k}}-\omega_{21} / 2-\Delta-i \lambda_{2}\right) t}-1\right],
\end{aligned}
$$

which in the limit $t \rightarrow \infty$ yields 


$$
\begin{aligned}
b_{\mathbf{k}}(\infty)= & g_{\mathbf{k} 1}\left[\frac{C_{1}}{\delta_{\mathbf{k}}+\frac{\omega_{21}}{2}-i \lambda_{1}}+\frac{C_{2}}{\delta_{\mathbf{k}}+\frac{\omega_{21}}{2}-i \lambda_{2}}\right] \\
& +g_{\mathbf{k} 2}\left[\frac{C_{1}^{\prime}}{\delta_{\mathbf{k}}-\frac{\omega_{21}}{2}-\Delta-i \lambda_{1}}+\frac{C_{2}^{\prime}}{\delta_{\mathbf{k}}-\frac{\omega_{21}}{2}-\Delta-i \lambda_{2}}\right] .
\end{aligned}
$$

The spontaneous emission spectrum $S\left(\delta_{\mathbf{k}}\right)$ in this model will be proportional to $\left|b_{\mathbf{k}}(\infty)\right|^{2}[14]$,

$$
\begin{aligned}
S\left(\delta_{\mathbf{k}}\right) \sim \gamma_{1}\left|\frac{C_{1}}{\delta_{\mathbf{k}}+\frac{\omega_{21}}{2}-i \lambda_{1}}+\frac{C_{2}}{\delta_{\mathbf{k}}+\frac{\omega_{21}}{2}-i \lambda_{2}}\right|^{2} \\
+\gamma_{2}\left|\frac{C_{1}^{\prime}}{\delta_{\mathbf{k}}-\frac{\omega_{21}}{2}-\Delta-i \lambda_{1}}+\frac{C_{2}^{\prime}}{\delta_{\mathbf{k}}-\frac{\omega_{21}}{2}-\Delta-i \lambda_{2}}\right|^{2} .
\end{aligned}
$$

By inspection of formula (24) we identify various sources of quantum interference that we would like to associate with the additional indistinguishable paths shown in Fig. 2. We consider in Fig. 2(a) the direct spontaneous emission from, for example, the lower state $|1\rangle$. Even with $p=\gamma_{2}=\cos \theta$ $=0$, an expansion in $\Omega^{2}$ of the spectrum would give rise to extra paths due to combined absorption and emission of stimulated driving field photons, where the leading term is depicted in Fig. 2(b1). In fact, the $\Omega^{2}$ terms in $\lambda_{1,2}$ should be read as $\Omega e^{\mathrm{i} \phi_{c}} \Omega e^{-\mathrm{i} \phi_{c}}$, showing that here an absorption of a microwave photon is always associated with the subsequent emission of another, canceling the phase dependence. Furthermore, for $\cos \theta \neq 0$ and thus the initial population also in the upper excited state, we obtain new phase-dependent terms in $C_{1}$ and $C_{2}$ corresponding to interfering paths as depicted in Fig. 2(b2). Here the path differs from that in Fig. 2(a) only by a single photon of the driving field, thus explaining the phase dependence.

For $p \neq 0$ we should return to the equations of motion (8), (11), and (12) and study numerically the behavior of the system. Then the most complex additional phase-dependent interference arises, which is associated with paths involving indistinguishable spontaneous photons from different transitions as depicted in Figs. 2(a) and 2(b3). This interference becomes maximal if both transitions to the ground state are exactly parallel and as we will see later when both levels are closely spaced, i.e., photons arising from both transitions become essentially indistinguishable. This type of interference has drawn much attention recently both for its effects on spontaneous emission dynamics of multilevel atoms $[11,13-$ $16]$ and also for its effects on the absorption, dispersion, and population dynamics of these atoms [21-23]. Actually, it is this type of interference that leads to the phase dependence in the microwave-driven $\Lambda$-type system studied by Martinez et al. [18] as happens in our scheme if the system is initially in one of the two excited states (either $\sin \theta$ or $\cos \theta=1$ ). If our system is furthermore initially prepared in a superposition of states $|1\rangle$ and $|2\rangle(\sin \theta, \cos \theta \neq 1)$, then phase-

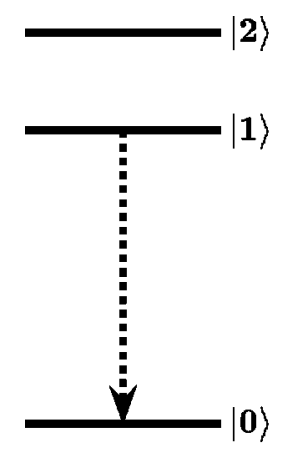

(a)

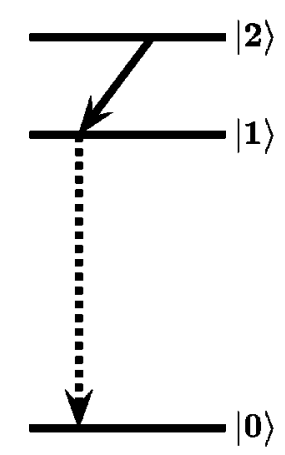

(b2)

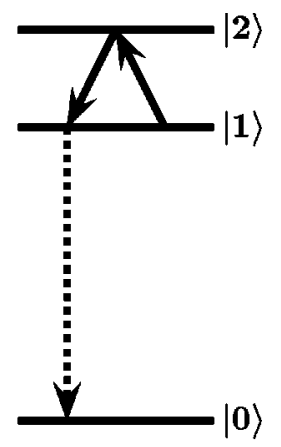

(b1)

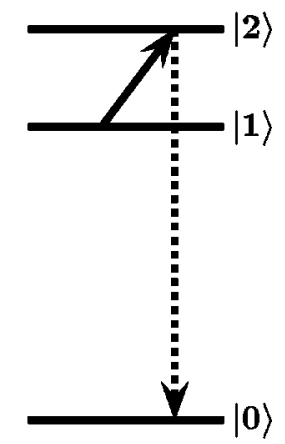

(b3)
FIG. 2. Leading interfering paths for the decay process from, e.g., the lower excited state; with solid lines we present the pathways for the driving field transitions and with dashed lines the spontaneous emission transitions. (a) Simple spontaneous decay. (b1) Phase-independent combined absorptions and emissions of driving field photons via the higher excited state. (b2) Phasedependent interfering paths for initial coherent superpositions via single stimulated emission from the higher-excited-state prior spontaneous emission. (b3) Phase-dependent single absorption of driving field photon followed by spontaneous emission from the higher excited state. In this case the paths are indistinguishable if the excited levels are closely spaced compared to the decay rates and if both dipole matrix elements coupling to the ground state are parallel.

dependent dynamics is observable even if $p=0$ as pointed out earlier, due to the processes of Figs. 2(a) and 2(b2).

\section{DISCUSSION}

We begin with an analysis of the system when one of the spontaneous decay rates is much smaller than the other and can be omitted, say, $\gamma_{2}=0$. This reduces our system to that studied by Knight [6] and Zhu et al. [12]. If we choose the atom to be initially in state $|1\rangle(\sin \theta=1, \cos \theta=0)$, then quantum interference can lead to complete or partial cancellation of specific emission modes and to spectral narrowing of one of the two peaks of the spectrum. The leading interfering process for weak driving fields is depicted in Fig. 2(b1). The system in this situation, however, is not phase sensitive. This occurs because stimulated absorption of a driving field photon is associated with the subsequent stimulated emission of another photon, taking it back to the de- 
caying state and thus canceling the phase dependence.

We proceed now with the case of an initial superposition of excited states, in particular when $\Omega \ll \gamma_{1}, \theta=\pi / 4$, and $\Delta=0$. In this case we can derive the following formulas for our system:

$$
\begin{gathered}
\lambda_{1} \approx-\frac{\gamma_{1}}{2}+2 \frac{\Omega^{2}}{\gamma_{1}}, \quad \lambda_{2} \approx-2 \frac{\Omega^{2}}{\gamma_{1}}, \\
C_{1} \approx \frac{\sqrt{2}}{\gamma_{1}}\left[\frac{\gamma_{1}}{2}+i \Omega e^{i \delta \phi}\right], \quad C_{2} \approx-\frac{\sqrt{2}}{\gamma_{1}} i \Omega e^{i \delta \phi},
\end{gathered}
$$

and

$$
b_{\mathbf{k}}(\infty) \approx \frac{g_{\mathbf{k} 1}\left(\delta_{\mathbf{k}}+\frac{\omega_{21}}{2}+\Omega e^{i \delta \phi}\right)}{\sqrt{2}\left(\delta_{\mathbf{k}}+\frac{\omega_{21}}{2}+i \frac{\gamma_{1}}{2}-2 i \frac{\Omega^{2}}{\gamma_{1}}\right)\left(\delta_{\mathbf{k}}+\frac{\omega_{21}}{2}+2 i \frac{\Omega^{2}}{\gamma_{1}}\right)} .
$$

An interesting feature occurs for $\delta \phi=0(\delta \phi=\pi)$ since the spectrum becomes zero at $\delta_{\mathbf{k}}=-\omega_{21} / 2-\Omega\left(\delta_{\mathbf{k}}=-\omega_{21} / 2\right.$ $+\Omega)$. The form of the spectrum for this case is shown in Fig. 3. For the particular values of $\delta \phi=0$ and $\delta \phi=\pi$ the spectrum resembles a Fano-type form [24], as shown in Figs. 3(a) and 3(c). The zero disappears for other values of the phase $\delta \phi$, as, for example, for $\delta \phi=\pi / 2$, as shown in Fig. 3 (b) where the spectrum is simply a single Lorentzian peak. The existence of this interference in the spectrum is associated with additional interfering paths as indicated in Fig. 2(b2). Obviously the interferences need not be destructive for each choice of parameters, so that we have an exact zero in the spectrum only for particular choices in the phase $\delta \phi$. An equally intuitive way of understanding the interferences can be given in the dressed-state picture and a detailed explanation will be given in Sec. IV. A similar effect has been investigated by Coleman and Knight [25] in the related area of resonant two-photon ionization and by Agassi in the area of spontaneous emission of autoionizing states [26]. We note that the appearance of exact zeros in the fluorescence spectrum is very sensitive to the radiative emission of the other level $(|2\rangle)$. Then various interfering paths analogous to Figs. 2(a) and 2(b3) arise with decay from the upper excited state $(|2\rangle)$ and obviously those do not need to interfer destructively for the same parameters at the same frequency. For example, in Fig. 4 we present the results for the spontaneous emission spectrum of this atom, now assuming a very weak emission from level $|2\rangle$, i.e., $\gamma_{2} \neq 0$, but also $\gamma_{2} \ll \gamma_{1}$. We note that the exact zero noted earlier has disappeared for those parameters. In this case the spectrum will also depend on the value of $p$. With both decay channels open and with $p \neq 0$ we involve further interfering channels such as those presented in Fig. 2(b3). The dashed curve in Fig. 4 shows the results with $p=0$ using Eq. (24) and the solid curve the results with $p=1$ from a numerical solution of Eqs. (8), (11), and (12). For $p=0$ we can immediately see that even for a small $\gamma_{2}$ of $0.5 \Omega$, as shown in Fig. 4(a), the precise zero disappears and a minimum well above zero takes its place in the spectrum. This minimum is further lifted when the decay rate of the upper excited state increases, as displayed in Figs. 4(b) and 4(c). In addition, a second peak appears in the spectrum due

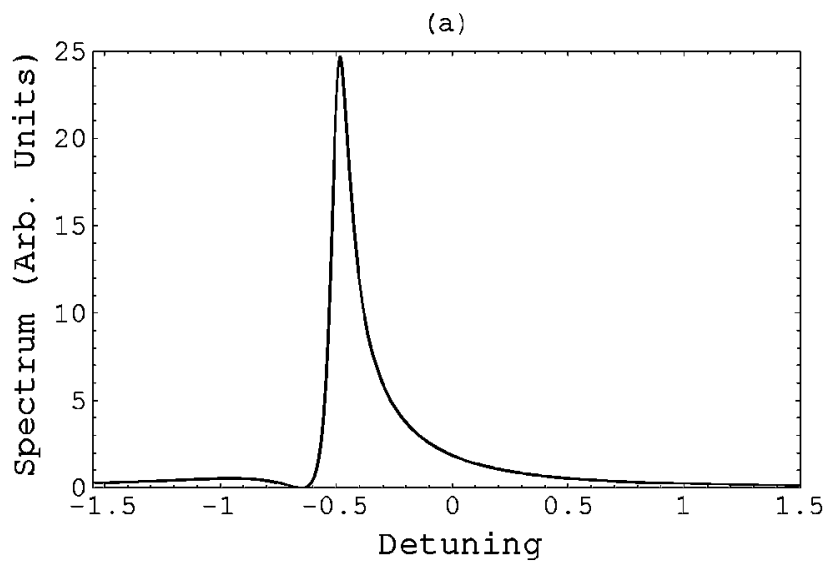

(b)

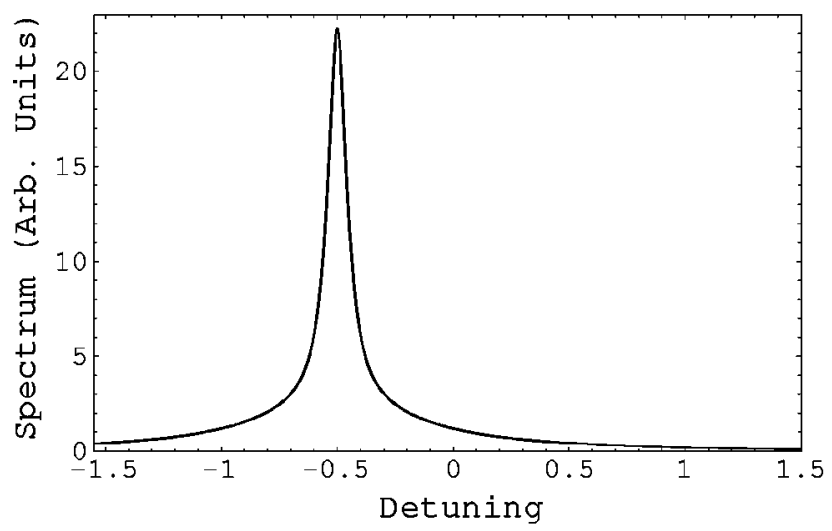

(c)

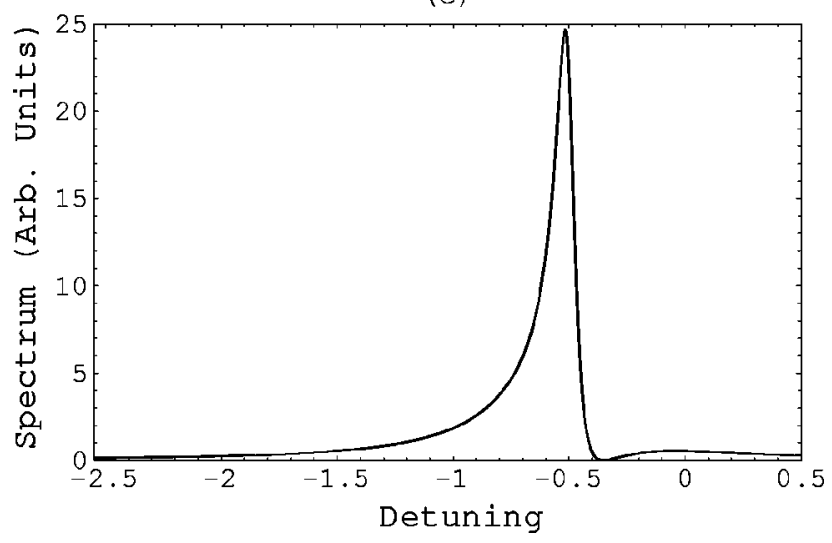

FIG. 3. Spontaneous emission spectrum $S\left(\delta_{\mathbf{k}}\right)$ (in arbitrary units) for atomic parameters $\gamma_{2}=0, \theta=\pi / 4, \Omega=0.15 \gamma_{1}, \Delta=0$, and $\omega_{21}=\gamma_{1}$. (a) $\delta \phi=0$, (b) $\delta \phi=0.5 \pi$, and (c) $\delta \phi=\pi$. The detuning $\delta_{\mathbf{k}}$ is measured in units of $\gamma_{1}$.

to the second decay rate. In the case that $p=1$ the behavior of the system is more complex due to the additional interfering channels originating from the mechanism displayed in Figs. 2(a) and 2(b3). First, we note specifically from Figs. 4(b) and 4(c) that the presence of this additional interference mechanism enhances the inhibition of spontaneous emission in the Fano minimum. Second, an additional zero appears in the spectrum that is solely associated with the new interference mechanism. This zero is quite stable to the increase of the decay rate $\gamma_{2}$. These results clearly show the coexistence of two distinguished mechanisms for fluorescence inhibition, which can act either independently or jointly in order to modify the spectrum. 
(a)

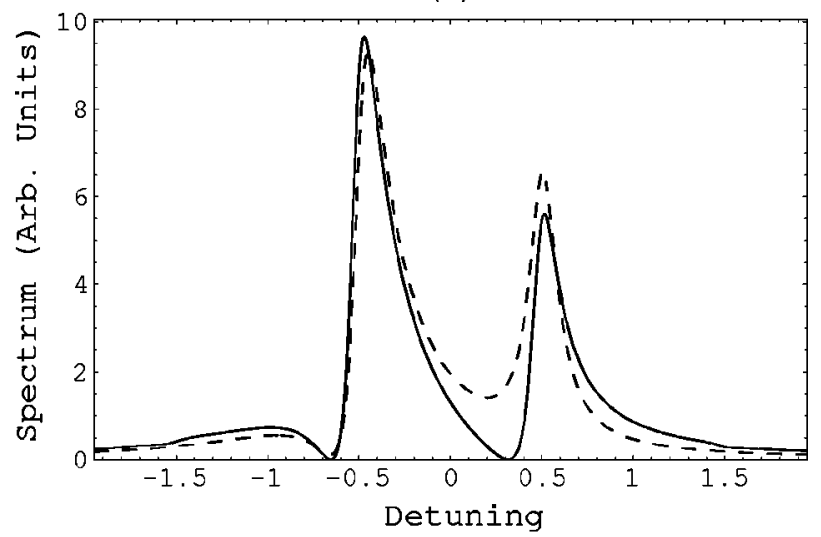

(b)

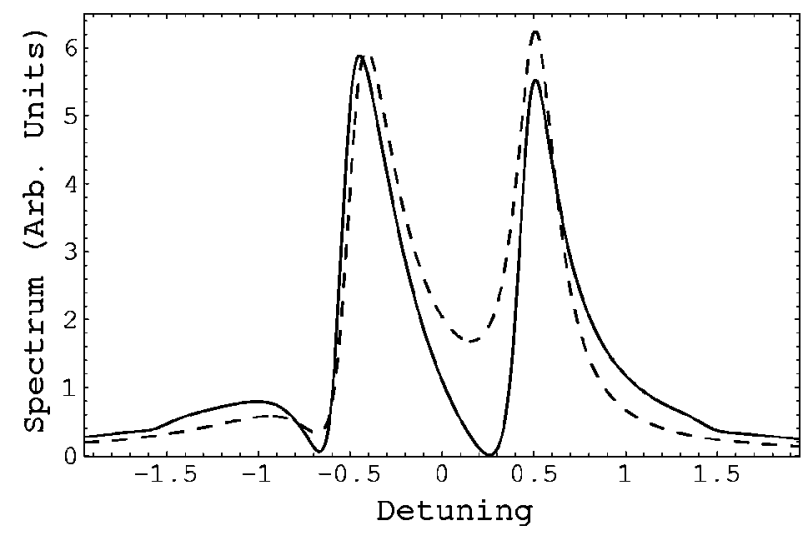

(c)

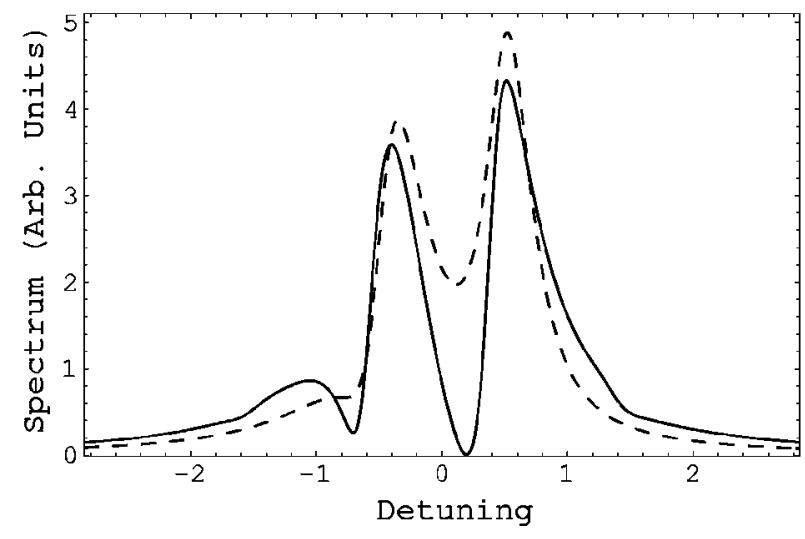

FIG. 4. Same as in Fig. 3(a), but for (a) $\gamma_{2}=0.075 \gamma_{1}=0.5 \Omega$, (b) $\gamma_{2}=0.15 \gamma_{1}=\Omega$, and (c) $\gamma_{2}=0.3 \gamma_{1}=2 \Omega$. By the dashed curve we present the results with $p=0$ and by the solid curve the results with $p=1$. The detuning $\delta_{\mathbf{k}}$ is measured in units of $\gamma_{1}$.

In the following we consider the case when $\gamma_{1}=\gamma_{2}=\gamma$ and $\Delta=0$ and examine the phase dependence of the system. We have seen from the previous discussion that the Fano interferences due to the decay channels $\gamma_{1}$ and $\gamma_{2}$ do not interfere destructively at the same frequency. Thus, for $\gamma_{1}$ $=\gamma_{2}$, we do not expect any zeros due to Fano interference and the other phase-dependent mechanism, which is displayed in Fig. 2(b3), will dominate. In this case the solutions (16) obtain the form

$$
\begin{aligned}
b_{1}(t)= & \frac{1}{2}\left[\left(\sin \theta-e^{i \delta \phi} \cos \theta\right) e^{-(\gamma / 2-i \Omega) t}\right. \\
& \left.+\left(\sin \theta+e^{i \delta \phi} \cos \theta\right) e^{-(\gamma / 2+i \Omega) t}\right],
\end{aligned}
$$

$$
\begin{aligned}
b_{2}(t)= & \frac{1}{2}\left[\left(\cos \theta-e^{-i \delta \phi} \sin \theta\right) e^{-(\gamma / 2-i \Omega) t}\right. \\
& \left.+\left(\cos \theta+e^{-i \delta \phi} \sin \theta\right) e^{-(\gamma / 2+i \Omega) t}\right]
\end{aligned}
$$

and finally Eq. (23) reads

$$
\begin{aligned}
b_{\mathbf{k}}(\infty)= & \frac{g_{\mathbf{k} 1}}{2}\left[\frac{\sin \theta-e^{i \delta \phi} \cos \theta}{\delta_{\mathbf{k}}+\frac{\omega_{21}}{2}+\Omega+i \frac{\gamma}{2}}+\frac{\sin \theta+e^{i \delta \phi} \cos \theta}{\delta_{\mathbf{k}}+\frac{\omega_{21}}{2}-\Omega+i \frac{\gamma}{2}}\right] \\
& +\frac{g_{\mathbf{k} 2}}{2}\left[\frac{\cos \theta-e^{-i \delta \phi} \sin \theta}{\delta_{\mathbf{k}}-\frac{\omega_{21}}{2}-\Delta+\Omega+i \frac{\gamma}{2}}\right. \\
& \left.+\frac{\cos \theta+e^{-i \delta \phi} \sin \theta}{\delta_{\mathbf{k}}-\frac{\omega_{21}}{2}-\Delta-\Omega+i \frac{\gamma}{2}}\right] .
\end{aligned}
$$

The case when $\sin \theta=\cos \theta=1 / \sqrt{2}$ is once more very interesting. When $\delta \phi=0$ Eqs. (28) and (29) take the simple form $P_{m}(t)=\left|b_{m}(t)\right|^{2}=\frac{1}{2} e^{-\gamma t}, m=1,2$. This indicates a simple exponentially decaying behavior of the populations. The same result is obtained for $\delta \phi=\pi$. For $\delta \phi=0.5 \pi$ the populations become $P_{m}(t)=\frac{1}{2} e^{-\gamma t}[1 \pm \sin (2 \Omega t)], m=1,2$. In this case populations of each state show decaying Rabi oscillations, but we should note that the total population just decays exponentially as in the case that $\delta \phi=0, \pi$. The spontaneous emission spectrum behavior displays very rich features. In Fig. 5 we present the results for the spontaneous emission spectrum for several values of the phase $\delta \phi$ and for the cases of both $p=0$ (shown by dashed curves) and $p=1$ (shown by solid curves). The spectrum is clearly double peaked for $\delta \phi=0$, as shown in Fig. 5(a), and for $\delta \phi=\pi$, shown in Fig. 5(e). It is easy to verify from Eqs. (24) and (30) that, in the case $p=0$, the spectrum for these atomic parameters and phase values is given by the sum of two Lorentzian curves. This is related to the occupation of just a single dressed state and will be analyzed later. However, for any other value between 0 and $\pi$ of the relative phase $\delta \phi$ the emission spectrum is made up of four peaks (two associated with each bare-state contribution for each of the two dressed states), as shown in Figs. 5(b)-5(d). For $p=1$ we note destructive or constructive interference between the two bare-state contributions from each dressed state, similar to that observed in the $\Lambda$ system [18]. Total line elimination cannot arise due to this interference mechanism. This is only possible for an appropriate initial preparation in one of the dressed states and decoupling to the other and naturally but trivially occurs if one of the bare channels is forbidden.

\section{DRESSED-STATE ANALYSIS}

The dressed-state approach of dynamics [27] is a very useful tool for further understanding the behavior of the system as presented in the preceding section. The diagonalization of the field-atom interaction Hamiltonian (in a rotating frame)

$$
H_{\text {field }}^{\prime}=-\Delta|2\rangle\left\langle 2\left|+\Omega_{c} e^{i \phi_{c}}\right| 1\right\rangle\left\langle 2\left|+\Omega_{c} e^{-i \phi_{c}}\right| 2\right\rangle\langle 1|
$$


(a)

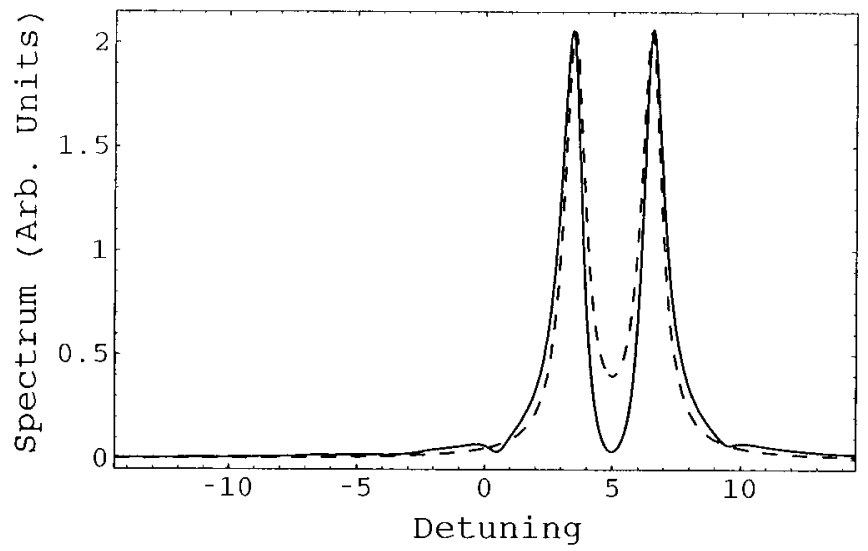

(c)

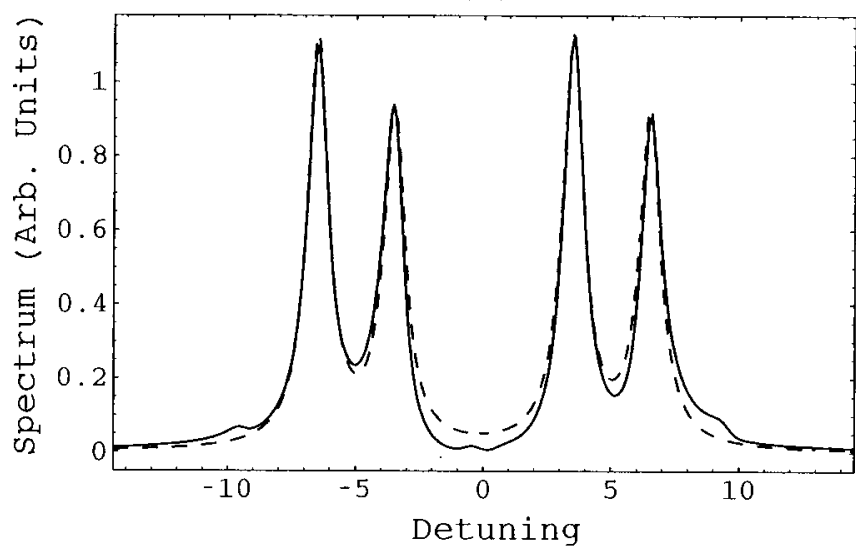

(b)

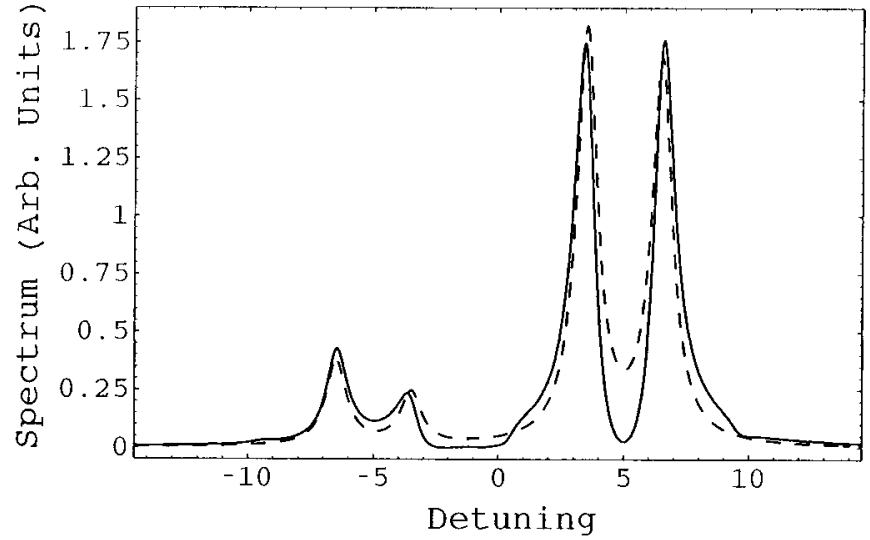

(d)

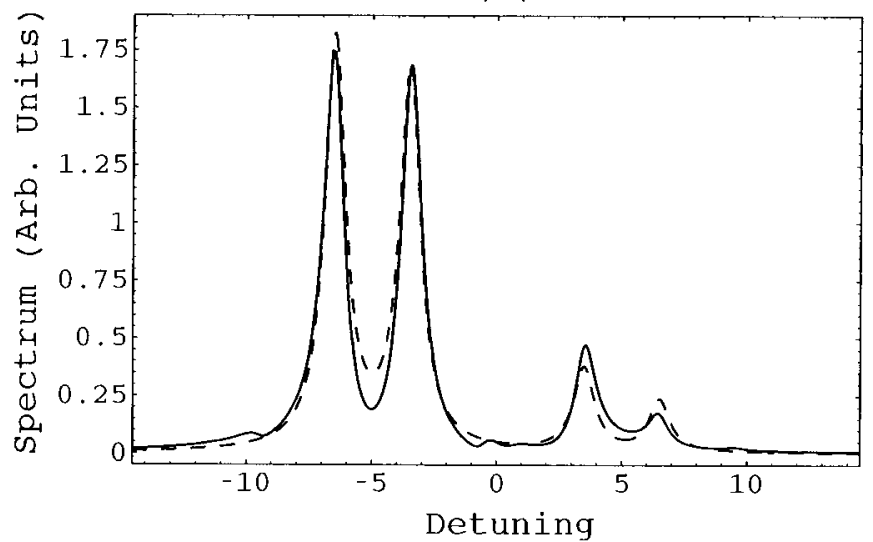

(e)

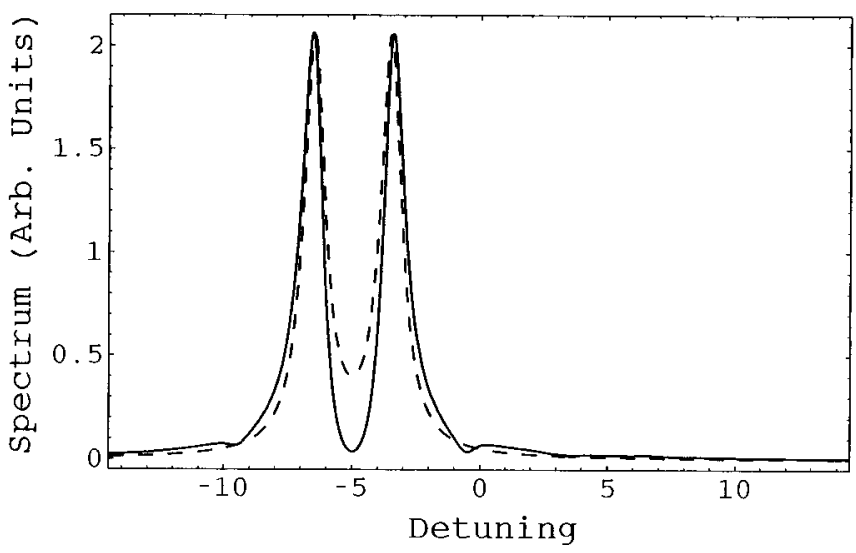

FIG. 5. Spontaneous emission spectra $S\left(\delta_{\mathbf{k}}\right)$ (in arbitrary units) for atomic parameters $\gamma_{2}=\gamma_{1}, \Omega=5 \gamma_{1}, \Delta=0$, and $\omega_{21}=3 \gamma_{1}$. (a) $\delta \phi=0$, (b) $\delta \phi=0.25 \pi$, (c) $\delta \phi=0.5 \pi$, (d) $\delta \phi=0.75 \pi$, and (e) $\delta \phi=\pi$. By the dashed curve we present the results with $p=0$ and by the solid curve the results with $p=1$. The detuning $\delta_{\mathbf{k}}$ is measured in units of $\gamma_{1}$.

leads to the dressed eigenstates

$$
\begin{aligned}
& |+\rangle=e^{-i \phi_{c}} \cos \Psi|1\rangle+\sin \Psi|2\rangle, \\
& |-\rangle=-\sin \Psi|1\rangle+e^{i \phi_{c} \cos \Psi|2\rangle,}
\end{aligned}
$$

where $\tan \Psi=E_{-} / \Omega$. Here $E_{ \pm} \equiv\left(-\Delta \pm \sqrt{\Delta^{2}+4 \Omega^{2}}\right) / 2$ are the eigenenergies of the Hamiltonian (31). The wave function of the system at time $t$ can be expressed in terms of the dressed states as

$$
\begin{aligned}
|\psi(t)\rangle= & b_{+}(t) e^{-i E_{+} t}|+,\{0\}\rangle+b_{-}(t) e^{-i E_{-} t}|-,\{0\}\rangle \\
& +\sum_{\mathbf{k}} b_{\mathbf{k}}(t) e^{-i \omega_{\mathbf{k}} t}|0,\{\mathbf{k}\}\rangle .
\end{aligned}
$$

The equations of motion of these amplitudes are given by

$$
\begin{gathered}
\dot{b}_{+}(t)=-\frac{1}{2} \gamma_{+} b_{+}(t)-\frac{1}{2} \gamma_{ \pm} b_{-}(t) e^{i E_{ \pm}^{\prime} t}, \\
\dot{b}_{-}(t)=-\frac{1}{2} \gamma_{-} b_{-}(t)-\frac{1}{2} \gamma_{\mp} b_{+}(t) e^{-i E_{ \pm}^{\prime} t} .
\end{gathered}
$$


Here $\gamma_{+}\left(\gamma_{-}\right)$is the $|+\rangle(|-\rangle)$dressed state decay to the ground state and $\gamma_{\mp}, \gamma_{ \pm}$are relaxations among the dressed states, as it is shown in Fig. 1(b). Also $E_{ \pm}^{\prime}=E_{+}-E_{-}$is the energy difference of the dressed states.

The probability amplitudes of the dressed states are connected to the probability amplitudes of the bare states by the relations

$$
\begin{gathered}
b_{+}(t)=e^{i \phi_{c}} \cos \Psi b_{1}(t)+\sin \Psi b_{2}(t), \\
b_{-}(t)=-\sin \Psi b_{1}(t)+e^{-i \phi_{c} \cos \Psi b_{2}(t) .}
\end{gathered}
$$

Using the initial condition (1), Eqs. (36) will reduce to

$$
\begin{gathered}
b_{+}(t=0)=e^{i \delta \phi} \cos \Psi \sin \theta+\sin \Psi \cos \theta, \\
b_{-}(t=0)=e^{-i \delta \phi}\left[-e^{i \delta \phi} \sin \Psi \sin \theta+\cos \Psi \cos \theta\right],
\end{gathered}
$$

which for the resonance case $(\Delta=0)$ yields

$$
\begin{gathered}
b_{+}(t=0)=\frac{1}{\sqrt{2}}\left(e^{i \delta \phi} \sin \theta-\cos \theta\right), \\
b_{-}(t=0)=\frac{e^{-i \delta \phi}}{\sqrt{2}}\left[e^{i \delta \phi} \sin \theta+\cos \theta\right] .
\end{gathered}
$$

So if $\theta=\pi / 4$ and $\delta \phi=0(\delta \phi=\pi)$ only the $|-\rangle(|+\rangle)$ dressed state is populated initially. In the weak-field limit and on resonance, the positions of the two dressed states are close in energy $E_{ \pm}^{\prime} \approx 0$ and the two dressed states are efficiently coupled to each other via the corresponding transitions to the ground state. Such a coupling is usually referred to as the off-diagonal coupling of the dressed states [25]. In the specific case that one of the two bare excited states decays $\left(\gamma_{2}=0\right)$, the dressed decay rates read

$$
\begin{gathered}
\gamma_{+}=\gamma_{-}=\frac{\gamma_{1}}{2}, \\
\gamma_{ \pm}=\gamma_{\mp}=\sqrt{\gamma_{+} \gamma_{-}}=\frac{\gamma_{1}}{2} .
\end{gathered}
$$

If the system is initially in one of the dressed states [the $|-\rangle$ dressed state in the case of Fig. 3(a) and the $|+\rangle$ dressed state in the case of Fig. 3(c)], then the population is efficiently transferred to the other dressed state due to this coupling via the ground state. This opens new channels for spontaneous emission and consequently interference structures in the spectrum, similar to a Fano-type behavior. For both states decaying transfer also occurs, but the actual forms of the dressed decay rates is far more complicated.

In the strong-field limit the energies of the dressed states differ substantially and the off-diagonal coupling is very weak, as the oscillating terms $e^{ \pm i E_{ \pm}^{\prime} t}$ become large. In this case, the two dressed states can be thought of as decaying independently [25]. Depending on the relative phase $\delta \phi$, either the positive or the negative frequency peaks of the spectrum can completely disappear, as can be seen in Figs. 5(a) and 5(e). This occurs as we can selectively populate one of the dressed states initially by appropriately choosing the phase difference $\delta \phi$ as explained by Eq. (39). This dressed state will subsequently decay without any transfer to the other dressed state and a two-peak structure may be created in the spectrum, where the two peaks are separated by the bare-state doublet spacing (given both bare states can decay). Even without selective dressed-state preparation there is no interference in the strong-field limit based on dressed offdiagonal couplings and extra paths due to dressed population transfer. This would furthermore be impossible because photons emitted from different dressed states become easily distinguishable if they are apart in frequency by more than the largest spontaneous emission rate. In spite of a large dressedstate separation, however, interference may still occur according to the mechanism described in Fig. 2(b3). This is because the two bare contributions of each dressed state then represent the different cornerstones for interfering paths rather than different dressed states, rendering only the spacing between the bare states relevant in this situation.

\section{CONCLUSIONS}

In this paper we have investigated the effects of the relative phase between a pump and a coupling field on the spontaneous emission spectrum from a three-level V-type system. We have shown that the modification of this phase difference allows us to efficiently control the shape of the spontaneous emission spectrum and the population dynamics. Various interference mechanisms were identified from the underlying transition physics. The various phase-dependent and -independent interferences responsible for the spectral structure depend sensitively on the relative energy and decay rates of the two excited states and on the orientation of the corresponding dipoles. In particular, we have shown that in the case in which only one excited state decays, the spontaneous emission spectrum can exhibit a Fano-type behavior in the weak-field limit and cancellation of the emission in a specific vacuum mode (i.e., specific radiated frequencies) occurs. In the dressed-state picture this originates from interferences involving indistinguishable photons due to an off-diagonal coupling between the dressed states. In this case the addition of decay to the other excited state could lead to more complicated sources of quantum interference. We have also emphasized that the dressed states decouple from each other for strong driving fields, yielding an inhibition of this source of interference. As a consequence of this decoupling, for specific initial preparation of solely one dressed state, we noted the inhibition of the total fluorescence corresponding to the other dressed state. This is controlled using the relative phase $\delta \phi$ that arises from the fact that the emission paths differ by an unequal number of stimulated absorptions and emissions of the microwave field photons. If the excited doublet states are closely spaced with respect to both decay rates and the corresponding emission dipoles are parallel, a different phase-dependent source of interference arises. This mechanism also survives in the strong-driving-field limit because it is not based on the coupling between the two dressed states as is the previous mechanism but on that of the bare-states contribution for each dressed state independently. In general, all these different sources of interference are present and compete, giving rise to a rich regime of parameters to shape the spectrum. 
An experimental realization of the phenomena discussed in this paper is possible using recent developments in quantum optics. For the first interference case, where the dipole moments of the two spontaneous emission transitions are orthogonal or the two upper states are well separated, the only essential part is the preparation of the excited doublet, as the atomic configuration can be realized in many atomic (or molecular) systems. This preparation can be achieved, for example, by pulses of specific area or by adiabatic transfer methods [28]. The second case is more restrictive in finding the proper atomic (or molecular) configuration as it requires the spontaneous emission matrix elements to be parallel and the quantum states close in energy. However, a similar configuration has been found in sodium dimers and already evidence of a related interference has been experimentally dem- onstrated [29]. Other possible experimental proposals include the use of atomic hydrogen [14,23]. Finally, it should be noted that this type of interference has been observed recently in tunneling transitions from semiconductor quantum wells $[30,31]$.

\section{ACKNOWLEDGMENTS}

We would like to thank M. B. Plenio for fruitful discussions. This work has been supported by the United Kingdom Engineering and Physical Sciences Research Council and the European Commission. C.H.K. has been funded by the Marie Curie scheme of the European Communilty and a "Nachwuchsgruppe" from the German Research Council (SFB 276).
[1] M. Lewenstein, T. W. Mossberg, and R. J. Glauber, Phys. Rev. Lett. 59, 775 (1987); C. H. Keitel, P. L. Knight, L. M. Narducci, and M. O. Scully, Opt. Commun. 118, 143 (1995); C. H. Keitel, J. Mod. Opt. 43, 1555 (1996); M. R. Ferguson, Z. Ficek, and B. J. Dalton, Phys. Rev. A 54, 2379 (1996); P. Zhou and S. Swain, ibid. 55, 772 (1997).

[2] S. Haroche and D. Kleppner, Phys. Today 42 (1), 24 (1989); B. M. Garraway and P. L. Knight, Phys. Rev. A 54, 3592 (1996).

[3] M. Lewenstein, J. Zakrzewski, and T. W. Mossberg, Phys. Rev. A 38, 808 (1988); S. John and T. Quang, ibid. 50, 1764 (1994); A. G. Kofman, G. Kurizki, and B. Sherman, J. Mod. Opt. 41, 353 (1994); S.-Y. Zhu, H. Chen, and H. Huang, Phys. Rev. Lett. 79, 205 (1997); S. Bay, P. Lambropoulos, and K. Mølmer, ibid. 79, 2654 (1997).

[4] G. S. Agarwal, in Quantum Statistical Theories of Spontaneous Emission and their Relation to Other Approaches, edited by G. Höhler et al., Springer Tracts in Modern Physics Vol. 70 (Springer-Verlag, Berlin, 1974).

[5] G. Alzetta, A. Gozzini, L. Moi, and G. Orriols, Nuovo Cimento B 36, 5 (1976); H. R. Gray, R. M. Whitley, and C. R. Stroud Jr., Opt. Lett. 6, 218 (1978); P. M. Radmore and P. L. Knight, J. Phys. B 15, 561 (1982); E. Arimondo, in Progress in Optics $X X X V$, edited by E. Wolf (Elsevier, Amsterdam, 1996), p. 257, and references therein.

[6] P. L. Knight, J. Phys. B 12, 3297 (1979); D. A. Cardimona, M. G. Raymer, and C. R. Stroud Jr., ibid. 15, 55 (1982).

[7] O. Kocharovskaya and Ya. I. Khanin, JETP Lett. 48, 630 (1988); S. E. Harris, Phys. Rev. Lett. 62, 1033 (1989); M. O. Scully, S.-Y. Zhu, and A. Gavrielides, ibid. 62, 2813 (1989); A. Imamoḡlu, Phys. Rev. A 40, 2835 (1989); G. S. Agarwal, Phys. Rev. Lett. 67, 980 (1991); M. Fleischhauer, C. H. Keitel, L. M. Narducci, M. O. Scully, S.-Y. Zhu, and M. S. Zubairy, Opt. Commun. 94, 599 (1992); C. H. Keitel, O. Kocharovskaya, L. M. Narducci, M. O. Scully, S.-Y. Zhu, and H. M. Doss, Phys. Rev. A 48, 3196 (1993).

[8] V. Vedral and M. B. Plenio, Prog. Quantum Electron. (to be published).

[9] L. M. Narducci, M. O. Scully, G. L. Oppo, P. Ru, and J. R. Tredicce, Phys. Rev. A 42, 1630 (1990); C. H. Keitel, L. M. Narducci, and M. O. Scully, Appl. Phys. B: Lasers Opt. 60,
S153 (1995); D. J. Gauthier, Y. Zhu, and T. W. Mossberg, Phys. Rev. Lett. 66, 2460 (1991).

[10] J. Javanainen, Europhys. Lett. 17, 407 (1992).

[11] G. C. Hegerfeldt and M. B. Plenio, Phys. Rev. A 46, 373 (1992); Quantum Opt. 6, 15 (1994).

[12] S.-Y. Zhu, L. M. Narducci, and M. O. Scully, Phys. Rev. A 52, 4791 (1995).

[13] P. Zhou and S. Swain, Phys. Rev. Lett. 77, 3995 (1996); Phys. Rev. A 56, 3011 (1997).

[14] S.-Y. Zhu, R. C. F. Chan, and C. P. Lee, Phys. Rev. A 52, 710 (1995); S. Y. Zhu and M. O. Scully, Phys. Rev. Lett. 76, 388 (1996); H. Huang, S.-Y. Zhu, and M. S. Zubairy, Phys. Rev. A 55, 744 (1997); H. Lee, P. Polynkin, M. O. Scully, and S.-Y. Zhu, ibid. 55, 4454 (1997); C. H. Keitel, S.-Y. Zhu, and P. L. Knight (unpublished).

[15] G. S. Agarwal, Phys. Rev. A 54, R3734 (1996); 55, 2457 (1997); A. K. Patnaik and G. S. Agarwal, J. Mod. Opt. 45, 2131 (1998).

[16] E. Paspalakis and P. L. Knight, Phys. Rev. Lett. 81, 293 (1998).

[17] For recent reviews see M. Shapiro and P. Brumer, J. Chem. Soc., Faraday Trans. 93, 1263 (1997); R. J. Gordon and S. A. Rice, Annu. Rev. Phys. Chem. 48, 601 (1997).

[18] M. A. G. Martinez, P. R. Herczfeld, C. Samuels, L. M. Narducci, and C. H. Keitel, Phys. Rev. A 55, 4483 (1997).

[19] T. Quang, M. Woldeyohannes, S. John, and G. S. Agarwal, Phys. Rev. Lett. 79, 5238 (1997).

[20] M. O. Scully, Phys. Rev. Lett. 55, 2802 (1985); M. O. Scully and M. S. Zubairy, Opt. Commun. 66, 303 (1988).

[21] E. Paspalakis, S.-Q. Gong, and P. L. Knight, Opt. Commun. 152, 293 (1998); S.-Q. Gong, E. Paspalakis, and P. L. Knight, J. Mod. Opt. (to be published).

[22] S. Menon and G. S. Agarwal, Phys. Rev. A 57, 4014 (1998).

[23] P. Zhou and S. Swain, Phys. Rev. Lett. 78, 832 (1997).

[24] U. Fano, Phys. Rev. 124, 1866 (1961).

[25] P. E. Coleman and P. L. Knight, J. Phys. B 14, 2139 (1981).

[26] D. Agassi, Phys. Rev. A 30, 2449 (1984). 
[27] C. Cohen-Tannoudji and S. Reynaud, in Multiphoton Processes, edited by J. H. Eberly and P. Lambropoulos (Wiley, New York, 1978).

[28] B. W. Shore, The Theory of Coherent Atomic Excitation Vols. $1 \& 2$ (Wiley Interscience, New York, 1990), and references therein.
[29] H.-R. Xia, C.-Y. Ye, and S.-Y. Zhu, Phys. Rev. Lett. 77, 1032 (1996).

[30] J. Faist, F. Capasso, C. Sirtori, K. W. West, and L. N. Pfeiffer, Nature (London) 390, 589 (1997).

[31] H. Schmidt, K. L. Campman, A. C. Gossard, and A. Imamoglu, Appl. Phys. Lett. 70, 3455 (1997). 